Check for updates

Cite this: RSC Adv., 2018, 8, 24477

\title{
Surface-enhanced Raman spectroscopic identification in fingerprints based on adhesive Au nanofilm $\uparrow$
}

\begin{abstract}
Jieru Lin, (D) Chenjie Zhang, Minmin Xu, Yaxian Yuan* and Jianlin Yao (D)*
The visualization and acquisition of information on substances within fingerprints have attracted considerable interest owing to their practical application in forensic science. There are still some challenges in the transfer and imaging of fingerprints and the extraction of residues. Here, a facile approach was successfully developed for transferring and recovering the pattern of fingerprints, which is based on surface-enhanced Raman spectroscopy (SERS) and an adhesive Au nanofilm (ANF). The reproducibility of SERS effects and the adhesive quality of the ANF enabled the transfer, recovery of the pattern and extraction of chemical residues from living/latent fingerprints. The results demonstrated that the pattern of living fingerprints, including ridges, furrows and sweat pores, was recovered on the basis of SERS mapping of the vibrational band of amino acids from endogenous protein substances. The dye rhodamine 6G (R6G) was employed as a developing agent to enhance the visualization of fingerprints by SERS mapping of the band at $1360 \mathrm{~cm}^{-1}$. Moreover, exogenous residues, such as cotinine (COT) and methylene blue (MB), were also detected by SERS. Their distribution in fingerprints was also determined, although it was not associated with the pattern of fingerprints. This indicated that the extraction process based on the adhesive ANF could be applied to transfer fingerprints from a crime scene to the laboratory for precise identification via structural information on chemical residues and the pattern image of fingerprints. It is anticipated that the adhesive ANF when combined with an ultrahigh-sensitivity SERS technique could be developed as a promising tool for the visualization of fingerprints and monitoring of trace chemical residues for crime tracking in forensic science.
\end{abstract}

Received 3rd May 2018

Accepted 1st June 2018

DOI: $10.1039 / c 8 \mathrm{ra03808b}$

rsc.li/rsc-advances
Currently, the acquisition and imaging of living fingerprints mainly rely on traditional methods such as marking on paper with an inkpad or using live scans. ${ }^{4,5}$ Such processes allow rapid visualization of the minutiae of fingerprints for identification. For latent fingerprint recognition, the use of specific chemical reagents or instruments enables the observation of colour or luminescence in the fingerprints, which provides an enhancement of the characteristic pattern of minutiae. ${ }^{1}$ Read-out technologies that correspond to these methods, including colorimetric $^{6,7}$ and fluorescence methods, ${ }^{8,9}$ mass spectrometry, ${ }^{10-12}$ infrared (IR) spectroscopy, ${ }^{13,14}$ localized surface plasmon resonance (LSPR), ${ }^{15}$ Raman spectroscopy ${ }^{16}$ and so on, are employed in latent fingerprint identification. Conventional colorimetric methods rely on changes in the colour of latent fingerprints that have been developed with materials such as magnetic powders, ${ }^{17}$ dyes, ${ }^{18}$ ninhydrin ${ }^{19}$ and cyanoacrylates ${ }^{20}$ via chemical or physical reactions. Fluorescence methods rely on inherent fluorescence or additional materials such as dyes, ${ }^{21}$ fluorescent quantum dots (QDs), ${ }^{8}$ immunolabelled nanoparticles $^{22,23}$ and metal or polymer nanoparticles.9,24 Most of these methods can be applied to a wide range of substrates but can only improve the patterns rather than extracting chemical information contained in latent fingerprints. However,
College of Chemistry, Chemical Engineering and Materials Science, Soochow University, Suzhou 215123, China. E-mail: yuanyaxian@suda.edu.cn; jlyao@suda. edu.cn

$\dagger$ Electronic supplementary information (ESI) available. See DOI: 10.1039/c8ra03808b 
chemical information involving sweat and its components and residues of explosives, drugs and chemicals is usually of great importance in crime tracking. Owing to the diversity of fingerprint components, it still remains a great challenge to recover such chemical information by rapid visualization and imaging. Recently, great efforts have been made to extract chemical information contained in fingerprints. Thus far, new read-out techniques, for instance, mass spectrometry and vibrational spectroscopy, including Raman and IR spectroscopy, have been successfully developed to overcome the drawbacks of traditional identification techniques, such as their time-consuming nature, lack of chemical information and low sensitivity. However, the abovementioned read-out techniques are still accompanied by significant disadvantages. For example, suppression of the ionization of analytes results in low sensitivity in mass spectrometric imaging. In vibrational spectroscopy, the trace water in fingerprints completely overwhelms the IR signals of chemical residues, and it is quite difficult to obtain Raman signals of trace residues owing to poor sensitivity. Furthermore, the exposure of latent fingerprints to different conditions results in difficulties in the visualization of fingerprints. Therefore, the development of a facile, non-destructive, highly efficient and low-cost analysis approach for fingerprint identification is highly desired, in particular for imaging the components of a fingerprint itself and enhancements in the visualization of latent fingerprints.

Surface-enhanced Raman spectroscopy (SERS) has been widely acknowledged to enhance conventional Raman signals by up to 14-15 orders of magnitude for adsorbed molecules. ${ }^{25-27}$ Two mechanisms, namely, electromagnetic field enhancement and chemical enhancement on the surface of molecules and nanoscale rough metals (mainly $\mathrm{Au}, \mathrm{Ag}$, and $\mathrm{Cu}$ ), are widely accepted. ${ }^{28,29}$ Currently, SERS detection has attracted considerable attention owing to the availability of portable instruments and flexible substrates. ${ }^{30-32}$ SERS detection technology has been applied in the identification of trace evidence, including document identification and the detection of explosives, biological systems, drugs or drug metabolites and so on. ${ }^{33-36}$ Moreover, SERS imaging techniques have wide-ranging applications in life sciences, such as monitoring the expression of anticancer drugs in living cells and the characterization of complex biomatrix biofilms. ${ }^{37,38}$ As a consequence, SERS has been developed as a promising approach for the identification of fingerprints owing to its ultrahigh sensitivity and abundant vibrational signatures, including the detection of components of residues $^{39-42}$ and visualization. ${ }^{43-45}$ Thus far, the applications of SERS in the detection of fingerprints can be divided into three categories: (i) the detection of endogenous and exogenous substances; (ii) visualization by SERS-based immunoassays or aptamer recognition; and (iii) direct visualization by SERS mapping. As examples of the first category, the chemical composition of eccrine sweat and trace drug-related biomarkers in fingerprints were detected by SERS techniques. ${ }^{39,40,46}$ Owing to the random distribution of the chemicals and the poor reproducibility of the SERS enhancements, SERS images and the patterns of fingerprints were not obtained in these studies. In the second category, on the basis of an immunoassay protocol Song et al. ${ }^{\mathbf{4 4}}$ developed an SERS imaging technique to detect different proteins within latent fingerprints. By the readout of SERS signals of Raman labels assembled on Ag immunonanoparticles, information on components was successfully resolved with high specificity and sensitivity. The pattern of latent fingerprints was then recovered on the basis of the different SERS responses of Raman labels used to modify Ag immunonanoparticles deposited on crossovers, furrows and ridge ends. Very similarly, the antibody was replaced by an aptamer for the visualization of latent fingerprints. Liu et al. ${ }^{\mathbf{4 5}}$ reported the high-resolution and universal visualization of latent fingerprints based on aptamer-functionalized nanoparticles with SERS probes. This approach exhibited several advantages, such as high specificity, comparable sensitivity, easy fabrication and no requirements for complicated treatments. It allowed us to identify various types of fingerprints on surfaces with different shapes. However, the immunoassay and aptamer recognition protocols were still time-intensive, and treatments in solution possibly caused the dissolution of endogenous or exogenous substances within the fingerprints, i.e., disappearance of the characteristic pattern of fingerprints. In the last category, a direct visualization method was developed on the basis of SERS mapping of trace chemical residues within fingerprints. A flexible and transparent SERS metafilm was fabricated by transferring a film of $\mathrm{Ag}$ nanocubes onto a tape, and the SERS-active transparent tape was used to cover the fingerprint for enhancing the Raman signals of residues in the fingerprint. ${ }^{43}$ The pattern of the fingerprint was recovered from the SERS intensities, which depended on the amounts of chemical residues in different ridges, furrows and crossovers. Moreover, the distribution of trace chemical residues was also measured using SERS spectral features accordingly. The flexibility of the SERS tape enabled imaging to be performed on irregular surfaces with reasonable generalizability. To a certain extent, SERS signals that originated from exogenous substances brought about a limitation on the types of fingerprints owing to the random distribution of residues. Connatser et al. ${ }^{41}$ developed an SERS imaging process that utilized the vibrational band due to $\mathrm{C}-\mathrm{H}$ bonds in sebaceous matter deposited onto an $\mathrm{Ag}$ elastomer substrate. However, most of the external organic chemical residues and endogenous components in the fingerprint contributed together to the SERS signal in this spectral region. As a result, the selectivity and ability to distinguish external residues and internal components in a fingerprint decreased dramatically. Therefore, on the basis of the above SERS studies of fingerprints, a direct approach for imaging the pattern of fingerprints is highly desired.

Here, a large-area adhesive and flexible SERS substrate based on Au nanofilm (ANF) was fabricated for imaging the pattern of living and latent fingerprints, together with the detection of chemical residues. ANF exhibits several advantages in comparison with conventional SERS substrates, including high enhancement factors, good reproducibility, cleanliness, durability, and flexibility, as well as adhesiveness. It allowed us to collect and transfer fingerprints for imaging via adhesive interactions. Imaging of living fingerprints was achieved using the human body's own proteins on the skin. Besides, 
rhodamine 6G (R6G) was selected as a developing agent to enhance the pattern of latent fingerprints. Moreover, narrow and high-resolution SERS bands were suitable for identifying residues and endogenous components. The application of ANF in fingerprint identification recovered the pattern of the fingerprint and determined complex residues in the fingerprint simultaneously. It provides a new approach for fingerprint identification in the field of criminal forensic science.

\section{Experimental}

\section{Materials}

Chloroauric acid tetrahydrate $\left(\mathrm{HAuCl}_{4} \cdot 4 \mathrm{H}_{2} \mathrm{O}\right)$, hydroxylamine hydrochloride $\left(\mathrm{NH}_{2} \mathrm{OH} \cdot \mathrm{HCl}\right)$, trisodium citrate dihydrate $\left(\mathrm{Na}_{3}\right.$ $\left.\mathrm{C}_{6} \mathrm{H}_{5} \mathrm{O}_{7} \cdot 2 \mathrm{H}_{2} \mathrm{O}\right)$, sulfuric acid $\left(\mathrm{H}_{2} \mathrm{SO}_{4}\right)$, hydrogen peroxide $\left(\mathrm{H}_{2} \mathrm{O}_{2}\right)$ and thiophenol (TP) were acquired from Sinopharm Chemical Reagent Co., Ltd., China. Polyvinylpyrrolidone (PVP, $M_{\mathrm{w}}=$ 10 000) was obtained from Acros Organics (USA). Methylene blue (MB) was bought from Shanghai Yuanye Biological Technology Co., Ltd., China. Rhodamine 6G (R6G) was bought from Sangon Biotech Co., Ltd., and cotinine (COT) was acquired from Toronto Research Chemicals. All solutions were prepared with Milli-Q water.

\section{Substrate preparation and fingerprint collection}

Preparation of ANF. Au nanoparticles with a diameter of $30 \mathrm{~nm}$ were prepared by a seed-mediated growth method ${ }^{47}$ using $15 \mathrm{~nm}$ Au nanoparticles as seeds, which were prepared by the Frens method. ${ }^{48} \mathrm{In}$ brief, $1 \mathrm{~mL}$ of $1 \%(\mathrm{w} / \mathrm{w})$ PVP and $1 \mathrm{~mL}$ of $1 \%(\mathrm{w} / \mathrm{w}) \mathrm{Na}_{3} \mathrm{C}_{6} \mathrm{H}_{5} \mathrm{O}_{7}$ were added to $25 \mathrm{~mL}$ of $15 \mathrm{~nm}$ Au seeds, followed by the addition of $20 \mathrm{~mL}$ of $25 \mathrm{mmol} \mathrm{L}{ }^{-1} \mathrm{NH}_{2} \mathrm{OH} \cdot \mathrm{HCl}$. Next, $20 \mathrm{~mL}$ of $0.1 \%(\mathrm{w} / \mathrm{w}) \mathrm{HAuCl}_{4} \cdot 4 \mathrm{H}_{2} \mathrm{O}$ was dropped into the

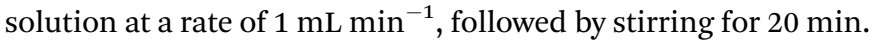
Then, the gas/liquid interfacial self-assembly technique was employed to form a large-area monolayer film of $\mathrm{Au}$ nanoparticles ( $\mathrm{Au} \mathrm{MLF}$ ) in a centrifuge tube after the solution was kept for $16 \mathrm{~h}$ at a temperature of $40{ }^{\circ} \mathrm{C} .{ }^{47}$ Finally, Au MLF was transferred to the adhesive side of tape to form ANF and was dried in ambient conditions prior to use.

Sampling procedure using ANF. TP was used as a model probe to evaluate the feasibility of ANF as an SERS substrate. Firstly, TP was fully modified on an SERS-active Au electrode, and then the electrode was rinsed with ethanol to remove excess molecules. The adhesive side of ANF was pressed continuously and tightly onto the Au electrode for 3 seconds and then peeled off for further measurements. This procedure allowed TP molecules to be transferred to ANF.

Living fingerprint collection. Living eccrine fingerprints were extracted with ANF. A clean fingertip was placed on ANF for 3 seconds, and then ANF was peeled off. Living fingerprints with external residues were prepared by the immersion of fingertips into a series of solutions and dried with $\mathrm{N}_{2}$. Similar collection procedures were performed for further SERS detection.

Latent fingerprint collection. Clean and contaminated latent fingerprints were both collected by pressing fingertips for a few seconds onto a cleaned object such as aluminum foil or glass.
The adhesive side of ANF evenly covered on the latent fingerprint and was then peeled off for further detection.

\section{Characterization}

Scanning electron microscopy (SEM) images of ANF were recorded by a Hitachi SU800 microscope. Transmission electron microscopy (TEM) images were acquired using a Tecnai F30 microscope with an accelerating voltage of $200 \mathrm{kV}$. All SERS spectra were acquired using an XploRA PLUS confocal microRaman system from Horiba equipped with a $638 \mathrm{~nm}$ laser with a power of about $0.2 \mathrm{~mW}$. The widths of the slit and pinhole were $300 \mu \mathrm{m}$ and $100 \mu \mathrm{m}$, respectively.

\section{Recovering the pattern of fingerprints}

Firstly, photographs of fingerprints were captured by a camera attached to the Raman spectrometer. Secondly, SERS mapping was performed on the living/latent fingerprint within the area of each photograph. The step for the accumulation of each SERS spectrum was about $40 \mu \mathrm{m}, 60$ spots were recorded in each direction ( $x$ or $y$ ), and in total 3600 SERS spectra were recorded in the selected area. The acquisition time for each spectrum was $10 \mathrm{~s}$. The SERS intensities of the characteristic bands due to internal components or external chemical residues were selected for the construction of SERS maps, and the pattern of the fingerprint was associated with the SERS mapping accordingly.

\section{Live subject statement}

All experiments were performed in accordance with the guidelines for human biomedical research from the Committee of Medical Ethics, the National Health Department of China, and the experiments were approved by the ethics committee at Soochow University. Informed consent was obtained from human participants in this study.

\section{Results and discussion}

\section{Fabrication and characterization of ANF}

Excellent monodispersity of the Au seeds and final $\mathrm{Au}$ nanoparticles was critical for the fabrication of ANF. This was further confirmed by a UV extinction spectrum, which showed a narrow absorption band (see Fig. S1A $\dagger$ ). Fig. 1A shows a TEM image of as-prepared Au MLF. It indicates that the mean diameter was about $30 \mathrm{~nm}$ and the Au nanoparticles were assembled in an orderly arrangement with a compact $2 \mathrm{D}$ structure. It was reasonable to assume that the small gaps between adjacent $\mathrm{Au}$ nanoparticles allowed the occurrence of a surface plasmon resonance (SPR) coupling effect, which contributed to a giant SERS effect and was defined in terms of "hot spots". After the transfer of Au MLF, an SEM image of ANF is shown in Fig. 1B, in which the substrate exhibits unique flatness and uniformity from an optical point of view.

Normal SERS substrates exhibited poor reproducibility owing to the random distribution of "hot spots". This definitely brought about significant difficulties in imaging surface compositions on the basis of SERS intensity. Therefore, the 


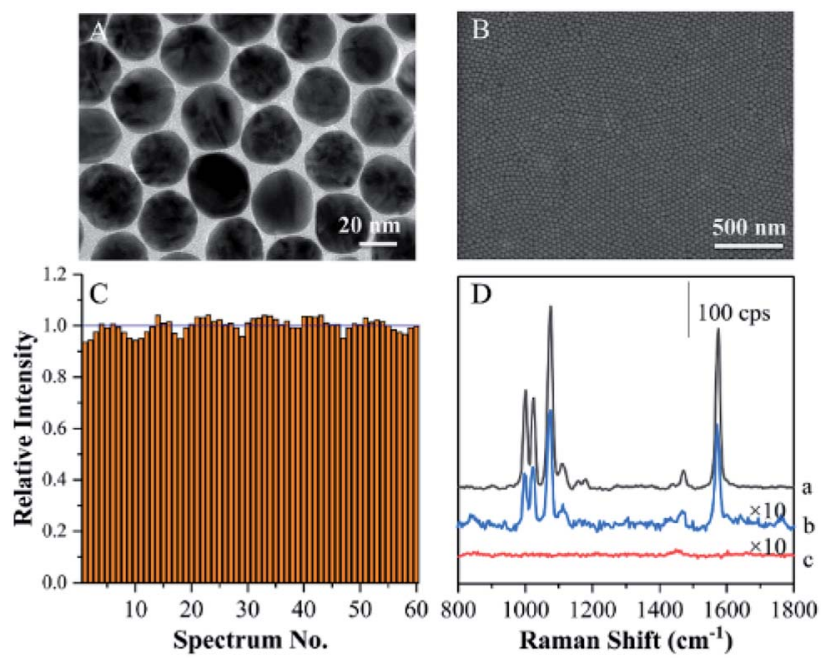

Fig. 1 (A) TEM image of Au MLF. (B) SEM image of ANF. (C) SERS intensities of TP at $1072 \mathrm{~cm}^{-1}$ relative to the average value at 60 randomly selected spots on ANF. (D) SERS spectra of TP adsorbed on a roughened Au electrode (a), ANF after the extraction of TP from the Au electrode (b), and blank ANF without extraction (c).

reproducibility of the SERS effect becomes a critical factor in recovering the pattern of fingerprints from the SERS intensities of endogenous and exogenous components. SERS mapping was performed on ANF using TP as a probe in a region of $20 \mu \mathrm{m} \times 20$ $\mu \mathrm{m}$. A series of SERS spectra were acquired from 60 spots within the selected region (as shown in Fig. S1B $\uparrow$ ). On comparing the average intensities at $1079 \mathrm{~cm}^{-1}$ of the 60 selected spectra, the variation in the intensity at $1079 \mathrm{~cm}^{-1}$ of each SERS spectrum was less than $10 \%$ (Fig. 1C). Therefore, this demonstrated that ANF exhibited excellent uniformity, because ordinary SERS substrates often exhibit differences in signal intensity of several orders of magnitude, which suggested that ANF fits the requirements for SERS mapping.

Actually, ANF could be used to attach and transfer a fingerprint or relevant sample. To demonstrate the sample collection procedure, ANF was used to cover a TP-modified roughened Au electrode and subsequently peeled off for SERS measurements. By comparing the SERS signals of the roughened Au electrode, ANF after sampling, and blank ANF, the characteristic SERS spectrum of TP was observed for the former two substrates and

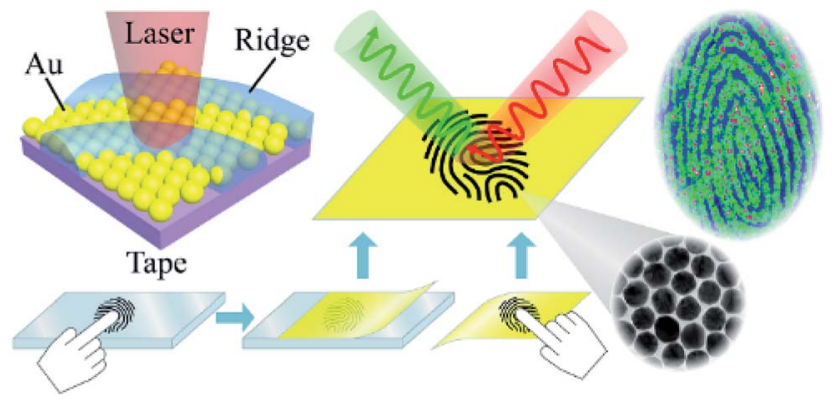

Fig. 2 Schematic diagram of the collection of living/latent fingerprints via the ANF transfer method and the process of SERS mapping of ANF. was absent for blank ANF (Fig. 1D). Although the SERS intensity was reduced remarkably after sampling from the roughened $\mathrm{Au}$ electrode, the features of the SERS spectra of ANF with adhered residues were easily recognizable for identifying the composition of the sampled surfaces. Therefore, this proved that the transfer of TP from an Au electrode to ANF was feasible and confirmed that the adhesiveness of tape played an important role in the collection and transfer of samples. On the basis of the above experimental facts, ANF is suitable for collecting and recovering the pattern of fingerprints (living and latent) from different kinds of surfaces.

\section{Living fingerprint capture with ANF}

The composition of human skin is complicated, as there are always quantities of lipids, proteins and amino acids. ${ }^{49}$ In addition, exogenous substances (residues) such as drugs, additives, and pesticides that originate from human social activity exist on the skin. It was expected to achieve both the extraction of target substances on a fingertip and SERS recognition based on the adhesive ANF. Two approaches were used to collect fingerprints: (i) living fingerprints were collected by placing the fingertip on ANF and (ii) latent fingerprints were collected by placing the adhesive ANF on fingerprints pressed onto different surfaces (as shown in Fig. 2). After the collection of the fingerprints, SERS measurements were performed to recover the pattern of the fingerprints on the basis of the SERS intensities of endogenous or exogenous substances within the fingerprints.

For living fingerprints, the adhesive side of ANF was employed for extracting the matrix on the fingertips, followed by SERS mapping. It was demonstrated that these fingerprint collection and SERS detection processes were convenient for in situ sample collection and the identification of information in various conditions without complex pretreatment and reactions.

We found that all fingerprints obtained from a living body by ANF had characteristic Raman spectra. Two dominant peaks at $1640 \mathrm{~cm}^{-1}$ and $1352 \mathrm{~cm}^{-1}$ were observed for the ridges of living fingerprints without exogenous substances (see Fig. S2 $\dagger$ ). Without the assistance of Au MLF or ANF, the obvious Raman bands of the fingerprints were absent. This suggested that the Raman signals of endogenous substances were effectively enhanced by the Au nanoparticles (as shown in Fig. S2 $\dagger$ ). Connatser et al. ${ }^{41}$ observed a similar band in the range of $1600-$ $1700 \mathrm{~cm}^{-1}$, which was assigned to the relevant vibrational mode of amino acids. Pelton $e t$ al. and Han et al. attributed the peak at $1640 \mathrm{~cm}^{-1}$ to a vibrational mode of proteins (amide I band). Therefore, it was believed that the peak at about $1640 \mathrm{~cm}^{-1}$ originated from endogenous substances within the fingerprint, and the pattern of living fingerprints could be recovered by SERS mapping of the band at $1640 \mathrm{~cm}^{-1}$. The significant difference in SERS intensities between ridges and furrows was used to demonstrate the different levels of detail and to recover the pattern lines of fingerprints. Fig. 3 shows an SERS map of a fingerprint based on the band at $1640 \mathrm{~cm}^{-1}$, together with an optical photograph captured by the camera. A representative 


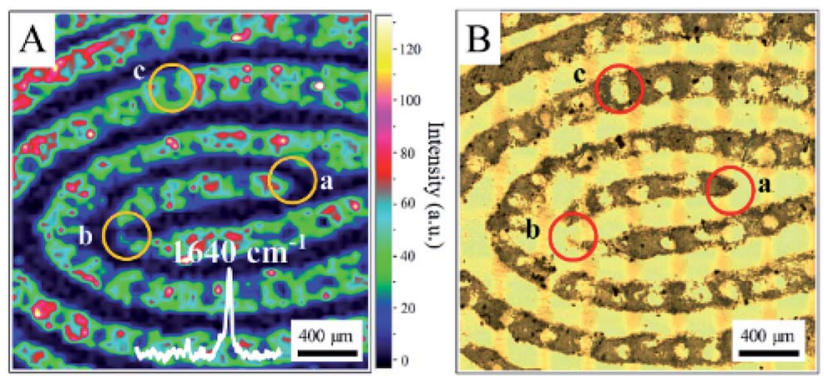

Fig. 3 (A) SERS map (1640 $\mathrm{cm}^{-1}$ ) of a living fingerprint based on endogenous substances captured by ANF. (B) Optical photograph of the living fingerprint on ANF.

spectrum acquired from a ridge of the living fingerprint is also shown in Fig. 3A. It should be pointed out that the acquisition time for each spectrum was $10 \mathrm{~s}$ and the total acquisition time was about 10 hours for high-quality SERS images. However, this was also carried out within 6 min with reasonable quality for rapid identification (see ESI Fig. S3†). The first-level details of lines of the fingerprint, including the lines of ridges and furrows, were clearly indicated by the SERS mapping. Secondlevel details, such as a termination point (marked as "a" in Fig. 3A) and an interrupt ("b" in Fig. 3A), were all well identified, as well as a pore ("c" in Fig. $3 \mathrm{~A}$ ) in the fingerprint (as shown in Fig. 3A). In the optical photograph, the gray part was assigned to the fingerprint ridge, and the corresponding position in the SERS map was the area of detectable signals. The bright area corresponded to furrow lines with an undetectable SERS signal. Therefore, on the basis of endogenous substances, the pattern of the fingerprint recovered from the SERS map closely coincided with the optical photograph (Fig. 3B). In addition, an image of the living fingerprint was recovered by SERS mapping after ANF was kept with the fingerprint in ambient conditions for 90 days, which indicated the reasonable stability of ANF and its ability to collect and store fingerprints.

\section{Identification of multiple components in living fingerprints}

In general, exogenous substances are always accompanied by endogenous lipids or proteins. The qualitative detection of exogenous substances is beneficial for recovering the pattern of a fingerprint from an SERS map and hence achieving crime tracking. Therefore, COT was selected as a model molecule to demonstrate the performance of ANF in determining the composition of residues within fingerprints. ${ }^{46} \mathrm{~A}$ fingertip was dipped into a $10^{-3} \mathrm{M}$ aqueous solution of COT, dried, and then pressed onto ANF. Two distinct SERS bands were observed unambiguously at $1030 \mathrm{~cm}^{-1}$ and $1640 \mathrm{~cm}^{-1}$, respectively (as shown in Fig. $\mathrm{S} 4 \mathrm{~A} \dagger)$. The band at $1030 \mathrm{~cm}^{-1}$ was attributed to the relevant vibrational mode of COT. SERS mapping was performed on the basis of the SERS intensities of COT at $1030 \mathrm{~cm}^{-1}$ and endogenous substances at $1640 \mathrm{~cm}^{-1}$, respectively (as shown in Fig. S4B and $\mathrm{D} \dagger$ ). The observation of very similar patterns of fingerprints indicated that COT was distributed on the fingerprint in the same way as the endogenous substances. Moreover, the pattern determined from both SERS bands was exactly fitted to an optical photograph of the fingerprint. Different amounts of COT in the different levels of detail of the fingerprint were associated with different intensities. Therefore, the pattern of the fingerprint was recovered successfully, accompanied by the ability to trace the composition of the exogenous residues. Unfortunately, although COT is considered to be a metabolite of nicotine, it was not detected on a smoker's fingerprint because COT was mainly present in the blood and urine rather than the skin sweat. This demonstrated that the performance of fingerprint imaging was improved significantly, and this could be developed into a promising approach in the recognition of fingerprints and crime tracking.

SERS has already been acknowledged to be a powerful technique for the characterization of molecules according to their characteristic peaks. Furthermore, the narrow and highresolution bands obtained by SERS mapping are promising for identifying and locating multiple components in fingerprints, because some residues did not always completely cover the fingerprint. SERS studies of two compounds present in fingerprints were performed to investigate the practicability of ANF in fingerprint identification. Here, we used R6G and MB as model compounds owing to their characteristic Raman bands with exact assignments. A fingertip was dipped into an ethanolic solution of R6G and dried. Subsequently, a small amount of an ethanolic solution of MB was spread on the fingertip and dried. The fingertip that contained R6G and MB was pressed onto ANF to leave the impression of a living fingerprint. The characteristic bands assigned to R6G and MB were observed unambiguously, i.e., at $1360 \mathrm{~cm}^{-1}$ for R6G and $1621 \mathrm{~cm}^{-1}$ for MB (as shown in Fig. 4A). Therefore, chemical information on multiple components in fingerprints could be clearly extracted. Furthermore, SERS mapping was performed using the bands of R6G at $1360 \mathrm{~cm}^{-1}$ and MB at $1621 \mathrm{~cm}^{-1}$, respectively (Fig. 4C
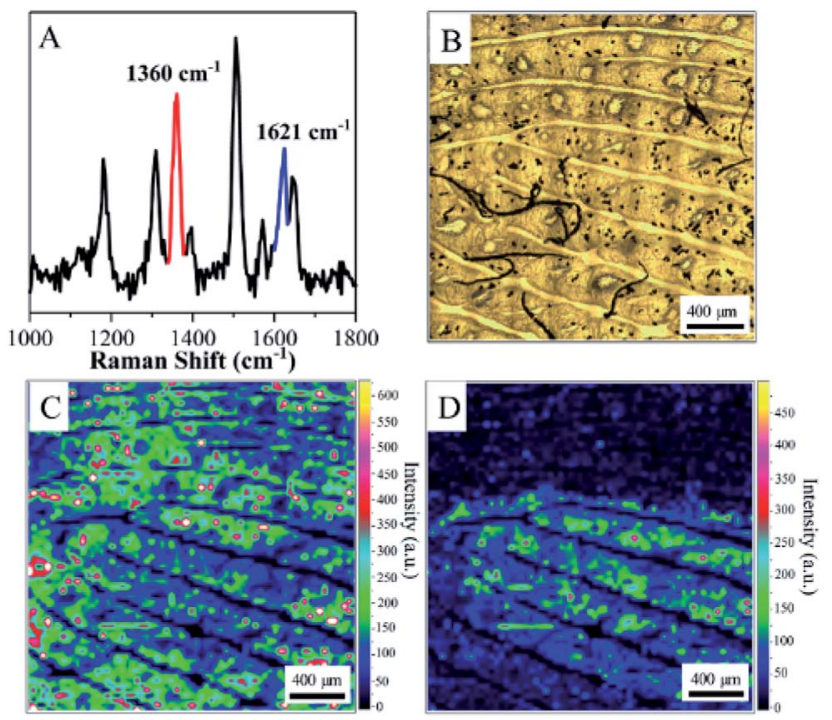

Fig. 4 (A) SERS spectrum acquired from the ridge of a fingerprint on ANF. (B) Optical image of the living fingerprint on ANF. SERS images of the living fingerprint visualized by R6G at $1360 \mathrm{~cm}^{-1}$ (C) and by MB at $1621 \mathrm{~cm}^{-1}$ (D). 
and $\mathrm{D})$. The pattern of the collected living fingerprint based on R6G molecules coincided with an optical image. This indicated that the distribution of R6G was associated with the details of the fingerprint. However, SERS mapping based on the band at $1621 \mathrm{~cm}^{-1}$ for MB indicated the pattern lines of fingerprints in the certain area that contained the residues. Although the whole pattern was not obtained, the composition of the residues was determined, which was considered to be direct evidence for crime tracking. In general, it was possible that Raman signals from the exogenous residues overwhelmed those from the endogenous substances, which resulted in difficulties in recovering the pattern of the fingerprint on the basis of the Raman signals of endogenous substances. Therefore, in the present case, the exogenous residue R6G played an important role in enhancing the pattern of fingerprints.

\section{Extraction and imaging of latent fingerprints with ANF}

The living fingerprint was always fresh, and the endogenous or exogenous substances that were left on ANF retained their original composition. However, the latent fingerprints were left on surfaces with different shapes and compositions, and the mixture of proteins, oil, sweat and other substances on the fingertip was transferred onto a solid surface accordingly. Moreover, exposure to air caused the fingerprints to age and their quantity and composition underwent changes, which resulted in difficulties in recovering the pattern of the latent fingerprints. Therefore, the extraction and imaging of latent fingerprints are highly significant for crime tracking in forensic sciences. Collection and transfer procedures were necessary prior to imaging, and highly sensitive techniques were required for detecting the composition of endogenous and exogenous substances. In the present case, the adhesive ANF was attached to the latent fingerprints by its sticky side and was gently peeled off the substrates for SERS detection (as shown in Fig. 2). This procedure allowed latent fingerprints to be transferred from a crime scene to the laboratory for precise measurements. Unfortunately, the amounts of trace endogenous and exogenous substances declined dramatically during the collection and transfer of the latent fingerprints. Although the obvious SERS signals of proteins at $1640 \mathrm{~cm}^{-1}$ were obtained from certain spots rather than all corresponding pattern lines, poor-quality fingerprint patterns were observed when the characteristic band was used for SERS mapping (as shown in Fig. S5†). Therefore, a dye was introduced to improve the quality of images as a developing agent. R6G is commonly used in fingerprint identification owing to its interaction with functional groups of residues and charged groups of amino acids in fingerprints and was considered as a model molecule for SERS investigations owing to the distinct assignments of its characteristic vibrational modes.

Therefore, a $10^{-4} \mathrm{M}$ aqueous solution of R6G was initially sprayed onto a latent fingerprint on a solid substrate and dried with $\mathrm{N}_{2}$. Secondly, the latent fingerprint modified with R6G was transferred to the adhesive ANF for SERS mapping (Fig. 5). The characteristic SERS spectral features of R6G, including the frequencies and relative intensities of the bands, were observed

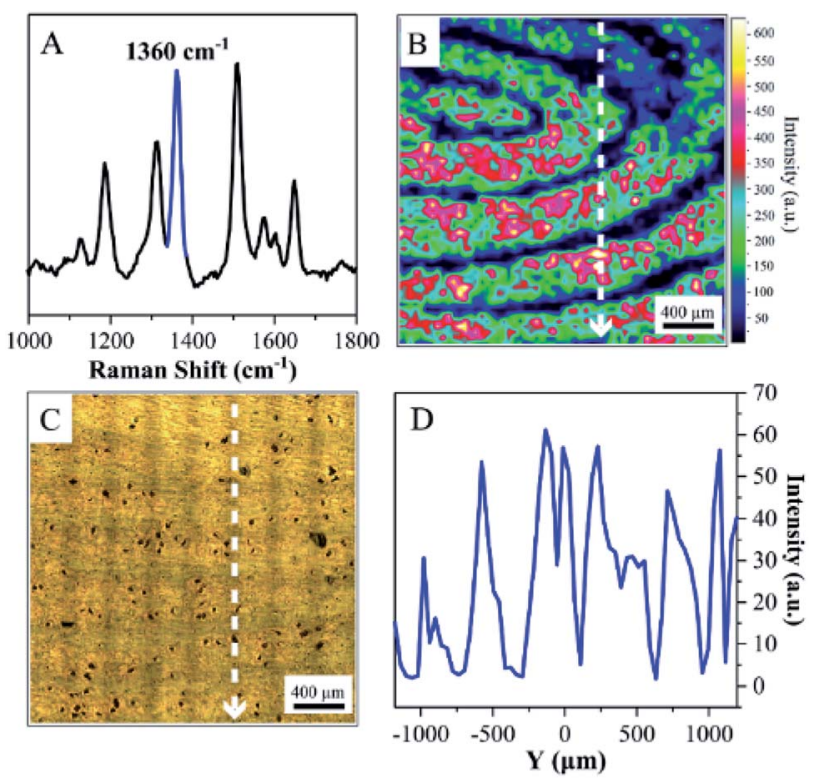

Fig. 5 (A) SERS spectrum of R6G acquired from ANF with a latent fingerprint. (B) SERS map of the latent fingerprint based on the band at $1360 \mathrm{~cm}^{-1}$. (C) Optical image of the latent fingerprint extracted by ANF. (D) SERS intensity-position profile of R6G at $1360 \mathrm{~cm}^{-1}$ along the $Y$-axis.

on the surface of the adhesive ANF (as shown in Fig. 5A). For this latent fingerprint, distinguishable details were not observed in its optical photograph (as shown in Fig. 5C). By comparison with the optical photograph, the unambiguous pattern of the fingerprint was recovered on the basis of the SERS intensities of the band at $1360 \mathrm{~cm}^{-1}$ (Fig. 5B). Positiondependent SERS intensities were determined along the $Y$-axis of the photograph of the fingerprint. Fig. 5D shows the distribution of the characteristic peak of R6G at $1360 \mathrm{~cm}^{-1}$ along the $Y$-axis, and the corresponding positions are shown in Fig. 5B and $\mathrm{C}$, respectively. This proved that the alternating maximum and minimum values of the SERS intensity were caused by the pattern lines of fingerprints, i.e., maxima for ridges and minima for furrows. This explanation was further confirmed by SERS mapping of the fingerprint after R6G was used as a developing agent. Therefore, the process of modification with R6G improved the efficiency of the visualization and collection of latent fingerprints.

\section{Identification of latent fingerprints with residues}

To further assess the ability of ANF to identify residues in latent fingerprints in real conditions, a volunteer was asked to dip a fingertip into a $10^{-3} \mathrm{M}$ aqueous solution of COT, which was dried and then pressed onto the surface of aluminium foil. A $10^{-4} \mathrm{M}$ aqueous solution of R6G served as a developing agent and was sprayed onto the latent fingerprint. Similarly, the latent fingerprint was collected and transferred to ANF. The representative spectrum displayed in Fig. 6A was acquired from ANF and exhibited the characteristic SERS spectral feature of R6G and the distinctive band of COT at $1030 \mathrm{~cm}^{-1}$. Actually, only 


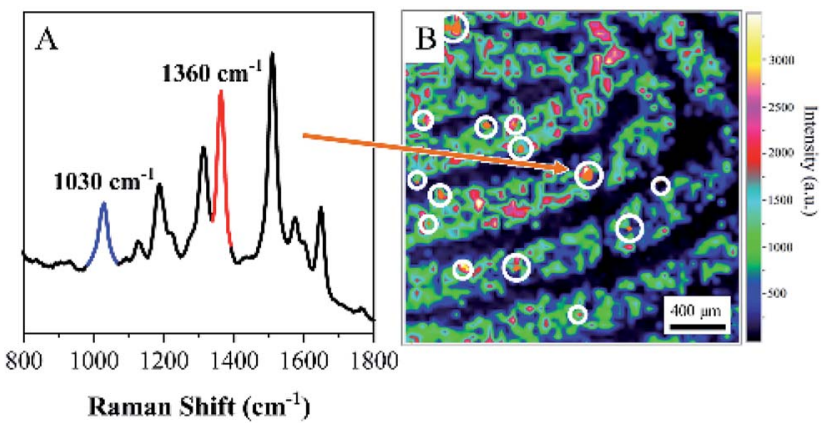

Fig. 6 (A) SERS spectrum acquired from ANF. (B) SERS image of latent fingerprint visualized by R6G using the band at $1360 \mathrm{~cm}^{-1}$.

a small amount of COT was left in the latent fingerprint; hence, COT could only be discovered in some locations, which indicated the presence of such residues in the latent fingerprint. However, owing to the interaction with endogenous substances, R6G was distributed in the fingerprint in accordance with its pattern. Fig. 6B shows the corresponding SERS map based on the SERS intensities of R6G at $1360 \mathrm{~cm}^{-1}$. Details of the fingerprint were demonstrated in the SERS imaging accordingly. As was mentioned above, the distribution of COT was not associated with the pattern of the fingerprint, and its SERS map lacked information on the details. After the SERS map of COT at $1030 \mathrm{~cm}^{-1}$ was overlapped with that of R6G at $1360 \mathrm{~cm}^{-1}$, the pattern of the fingerprints was visualized, together with information on exogenous residues. Owing to the wide spectral range of SERS, the rich spectral signature was exploited to achieve the detection of various exogenous residues. The technique based on ANF in combination with SERS could be developed as a promising approach for combining the multiple functions of sample collection, visualization and analysis of trace exogenous residues.

\section{Conclusions}

In summary, a non-invasive imaging technique was successfully developed for the in situ sampling and recovery of the pattern of fingerprints on the basis of SERS and the adhesive ANF. The assembly of large-scale Au MLF on adhesive tape brought about high SERS activity and reproducibility, as well as adhesiveness and flexibility. It enabled the collection and transfer of samples from different objects. SERS mapping was performed to visualize the pattern of fingerprints and monitor the distribution of chemical residues. The adhesive ANF collected two kinds of fingerprints, namely, living and latent fingerprints. For living fingerprints, the distribution of endogenous protein substances was used to form the pattern of the fingerprints on the basis of SERS mapping of the band at $1640 \mathrm{~cm}^{-1}$ assigned to the relevant vibrational mode of amino acids. The pattern lines of ridges and furrows were recovered unambiguously, together with the third-level details of sweat pores. The features of the pattern were closely associated with an optical image of the fingerprint. In order to improve the efficiency of the identification of latent fingerprints, the dye R6G was employed as a developing agent for enhancing the visualization of latent fingerprints on the basis of SERS mapping of the band at $1360 \mathrm{~cm}^{-1}$ assigned to the vibrational mode of R6G. Moreover, exogenous residues, such as COT and MB, were also detected by SERS mapping, although their distribution in fingerprints was not associated with the pattern. The results demonstrated that the extraction process using the adhesive ANF could be employed to collect fingerprints on different objects. It allowed us to transfer fingerprints from a crime scene to the laboratory for precise identification. We believe that the technique based on the adhesive ANF in combination with ultrahigh-sensitivity SERS could be developed as a promising tool for the visualization of fingerprints and monitoring of trace chemical residues for crime tracking in forensic science.

\section{Conflicts of interest}

There are no conflicts to declare.

\section{Acknowledgements}

The authors gratefully acknowledge the financial support received from the National Science Foundation of China (No.: 21673152, 21773166, 21473118). The partial financial support is from a project funded by the Priority Academic Program Development of Jiangsu Higher Education Institutions (PAPD) and the Project of Scientific and Technologic Infrastructure of Suzhou (SZS201708).

\section{Notes and references}

1 O. P. Jasuja, G. S. Bumbrah and R. M. Sharma, Research and Reports in Forensic Medical Science, 2016, 6, 39-50.

2 P. Hazarika and D. A. Russell, Angew. Chem. Int. Ed., 2012, 51, 3524-3531.

3 R. M. Sapstead, K. S. Ryder, C. Fullarton, M. Skoda, R. M. Dalgliesh, E. B. Watkins, C. Beebee, R. Barker, A. Glidle and A. R. Hillman, Faraday Discuss., 2013, 164, 391.

4 C. N. G. Sambasiva Rao, L. S. S. Reddy and E. V. Prasad, International Journal of Computer Science and Network Security, 2008, 8, 394-397.

5 S. T. V. Parthasaradhi, R. Derakhshani, L. A. Hornak and S. A. C. Schuckers, IEEE Trans. Syst. Man Cybern. Part C Appl. Rev., 2005, 35, 335-343.

6 J. Lee, M. Pyo, S. H. Lee, J. Kim, M. Ra, W. Y. Kim, B. J. Park, C. W. Lee and J. M. Kim, Nat. Commun., 2014, 5, 3736.

7 J. Dutta, S. A. Ramakrishna and I. Mekkaoui Alaoui, Forensic Sci. Int., 2013, 228, 32-37.

8 Y. J. Jin, Y. J. Luo, G. P. Li, J. Li, Y. F. Wang, R. Q. Yang and W. T. Lu, Forensic Sci. Int., 2008, 179, 34-38.

9 H. Chen, R. L. Ma, Y. Chen and L. J. Fan, ACS Appl. Mater. Interfaces, 2017, 9, 4908-4915.

10 D. R. Ifa, N. E. Manicke, A. L. Dill and G. Cooks, Science, 2008, 321, 805.

11 L. S. Ferguson, S. Creasey, R. Wolstenholme, M. R. Clench and S. Francese, J. Mass Spectrom., 2013, 48, 677-684. 
12 G. B. Yagnik, A. R. Korte and Y. J. Lee, J. Mass Spectrom., 2013, 48, 100-104.

13 A. V. Ewing and S. G. Kazarian, Analyst, 2017, 142, 257-272. 14 C. Ricci, P. Phiriyavityopas, N. Curum, K. L. A. Chan, S. Jickells and S. G. Kazarian, Appl. Spectrosc., 2007, 61, 514-522.

15 T. Peng, W. Qin, K. Wang, J. Shi, C. Fan and D. Li, Anal. Chem., 2015, 87, 9403-9407.

16 J. S. Day, H. G. Edwards, S. A. Dobrowski and A. M. Voice, Spectrochim. Acta, Part A, 2004, 60, 1725-1730.

17 G. S. Sodhi and J. Kaur, Forensic Sci. Int., 2001, 120, 172-176.

18 K. Braasch, M. de la Hunty, J. Deppe, X. Spindler, A. A. Cantu, P. Maynard, C. Lennard and C. Roux, Forensic Sci. Int., 2013, 230, 74-80.

19 D. B. Hansen and M. M. Joullie, Chem. Soc. Rev., 2005, 34, 408-417.

20 M. Takatsu, O. Shimoda and H. Teranishi, J. Forensic Sci., 2012, 57, 515-520.

21 X. Jin, R. Xin, S. Wang, W. Yin, T. Xu, Y. Jiang, X. Ji, L. Chen and J. Liu, Sens. Actuators, B, 2017, 244, 777-784.

22 P. Hazarika, S. M. Jickells, K. Wolff and D. A. Russell, Angew. Chem. Int. Ed., 2008, 47, 10167-10170.

23 C. M. Liu, L. Y. Zhang, L. Li, B. Y. Li, C. G. Wang and T. T. Wang, Dyes Pigm., 2018, 149, 822-829.

24 Y. H. Chen, S. Y. Kuo, W. K. Tsai, C. S. Ke, C. H. Liao, C. P. Chen, Y. T. Wang, H. W. Chen and Y. H. Chan, Anal. Chem., 2016, 88, 11616-11623.

25 M. Fleischmann, P. J. Hendra and A. J. McQuillan, Chem. Phys. Lett., 1974, 26, 163-166.

26 S. Nie, Science, 1997, 275, 1102-1106.

27 K. Kneipp, Y. Wang, H. Kneipp, L. T. Perelman, I. Itzkan, R. Dasari and M. S. Feld, Phys. Rev. Lett., 1997, 78, 16671670.

28 M. Moskovits, Rev. Mod. Phys., 1985, 57, 783-826.

29 J. Neugebauer, Phys. Rep., 2010, 489, 1-87.

30 A. Rousaki, C. Vázquez, V. Aldazábal, C. Bellelli, M. Carballido Calatayud, A. Hajduk, E. Vargas, O. Palacios, P. Vandenabeele and L. Moens, J. Raman Spectrosc., 2017, 48, 1459-1467.
31 L. Polavarapu and L. M. Liz-Marzan, Phys. Chem. Chem. Phys., 2013, 15, 5288-5300.

32 J. Chen, Y. Huang, P. Kannan, L. Zhang, Z. Lin, J. Zhang, T. Chen and L. Guo, Anal. Chem., 2016, 88, 2149-2155.

33 C. Muehlethaler, M. Leona and J. R. Lombardi, Anal. Chem., 2016, 88, 152-169.

34 A. Raza and B. Saha, Forensic Sci. Int., 2013, 233, 21-27.

35 A. Hakonen, P. O. Andersson, M. Stenbaek Schmidt, T. Rindzevicius and M. Kall, Anal. Chim. Acta, 2015, 893, 113.

36 K. V. Kong, Z. Lam, W. D. Goh, W. K. Leong and M. Olivo, Angew. Chem. Int. Ed., 2012, 51, 9796-9799.

37 W. Song, L. Ding, Y. Chen and H. Ju, Chem. Commun., 2016, 52, 10640-10643.

38 N. P. Ivleva, M. Wagner, A. Szkola, H. Horn, R. Niessner and C. Haisch, J. Phys. Chem. B, 2010, 114, 10184-10194.

39 J. Chen, G. Qin, Q. Chen, J. Yu, S. Li, F. Cao, B. Yang and Y. Ren, J. Mater. Chem. C, 2015, 3, 4933-4944.

40 Z. Gong, H. Du, F. Cheng, C. Wang, C. Wang and M. Fan, ACS Appl. Mater. Interfaces, 2014, 6, 21931-21937.

41 R. M. Connatser, S. M. Prokes, O. J. Glembocki, R. L. Schuler, C. W. Gardner, S. A. Lewis Sr and L. A. Lewis, J. Forensic Sci., 2010, 55, 1462-1470.

42 T. Yang, X. Guo, H. Wang, S. Fu, Y. Wen and H. Yang, Biosens. Bioelectron., 2015, 68, 350-357.

43 X. Liu, J. Wang, J. Wang, L. Tang and Y. Ying, Anal. Chem., 2016, 88, 6166-6173.

44 W. Song, Z. Mao, X. Liu, Y. Lu, Z. Li, B. Zhao and L. Lu, Nanoscale, 2012, 4, 2333-2338.

45 J. Zhao, K. Zhang, Y. Li, J. Ji and B. Liu, ACS Appl. Mater. Interfaces, 2016, 8, 14389-14395.

46 S. Han, S. Hong and X. Li, J. Colloid Interface Sci., 2013, 410, 74-80.

47 Q. Guo, M. Xu, Y. Yuan, R. Gu and J. Yao, Langmuir, 2016, 32, 4530-4537.

48 G. Frens, Nature, 1973, 241, 20-22.

49 C. Leordean, V. Canpean and S. Astilean, Spectrosc. Lett., 2012, 45, 550-555. 